\title{
The Impact of Acute Rejection in Kidney Transplantation on Long-Term Allograft and Patient Outcome
}

\author{
Mojgan Jalalzadeh ${ }^{1}$; Nouraddin Mousavinasab ${ }^{2}$; Said Peyrovi ${ }^{3}$; Mohammad Hassan \\ Ghadiani $^{4, *}$ \\ ${ }^{1}$ Department of Nephrology, Imam Hossein Hospital, Shahid Beheshti University of Medical Sciences, Tehran, IR Iran \\ ${ }^{2}$ Department of Social Medicine, Zanjan University of Medical Sciences, Zanjan, IR Iran \\ ${ }^{3}$ Maraghe University of Medical Sciences, Maraghe, IR Iran \\ ${ }^{4}$ Department of Nephrology, Taleghani Hospital, Shahid Beheshti University of Medical Sciences, Tehran, IR Iran \\ *Corresponding author: Mohammad Hassan Ghadiani, Department of Nephrology, Taleghani Hospital, Shahid Beheshti University of Medical Sciences, Tehran, IR Iran. Tel: +98- \\ 9122248306, E-mail: j_mojgan@yahoo.com
}

Received: October 10, 2014; Revised: November 1, 2014; Accepted: November 5, 2014

Background: Patients with end stage renal disease(ESRD) can be sustained with dialysis therapy.

Objectives: In this study, we followed up the effect of early acute and late acute rejections on survival rates of patients' grafts.

Patients and Methods: We investigated the timing and frequency of acute rejection episodes related to long-term patient-graft survival in Taleghani hospital between 1990 and 2011. Recipients were divided into three groups as Group-1 (no rejection), Group-2 (early acute rejection [EAR]: less than 3-months) and Group-3 (late acute rejection [LAR]: after 3 months of transplant).

Results: One and five-year patient's survival rates were $94.87 \%$ and $93.8 \%$, and graft survival (GS) rates were 92.6\% and $81.9 \%$. EAR and LAR occurred in 125 (18.8\%) and 77 (11.7\%) patients, respectively. Graft and patient survival rates at one and five years were as follows; Group-1(Graft 96.7\% and 94.5\% patient: 97.4\% and 96.8\%), Group-2 (Graft:72\% and 61\%, patient: $85.6 \%$ and $84 \%$ ), Group-3 (Graft: $84.4 \%$ and 36.8\%, patient: $92.2 \%$ and $89.4 \%$ ). Recipient age, type and length of dialysis, number of transplantations and the status of panel reactivity antibody (PRA) had no effect on the type of rejection. LAR was more commonly associated with males $(\mathrm{P}=0.001)$ and donors' age was associated with rejection $(\mathrm{P}$ $=0.0002)$. Five-year GS rate among the three groups was lower in the LAR group $(\mathrm{P}<0.0001)$.

Conclusions: LAR had a negative impact on long-term renal allograft survival and the risk of chronic graft dysfunction increased in patients with a history of LAR.

Keywords:Graft Survival; Rejection; Survival; Kidney Transplantation

\section{Background}

Patients with end stage renal disease (ESRD) can be sustained with dialysis therapy. However, patient's survival rate is higher with renal transplantation, which makes it the preferred method of treatment. In addition, renal transplant recipients have better quality of life than those under dialysis (1). However, long-term allograft survival is still limited. Many factors affect patient-graft survival in renal transplantation such as donor or recipient age, gender, race, primary cause of renal failure, cold ischemia time, HLA matching, prior transfusion, blood groups and preservation methods (2-5). Acute rejection (AR) is a common complication in renal transplantation and associated with reduced graft survival (6). It can occur less than three months (early acute rejection, EAR) or after three months of transplant (late acute rejection, LAR). While rejection episodes and delayed graft function (DGF) increase the risk of renal allograft loss (7, 8), strong immunosuppressive therapies reduce the frequency of acute rejection (9).

\section{Objectives}

The aim of this study was to investigate the timing of AR in a sample population and the relative impact of early and late AR on patients-grafts survival.

\section{Materials and Methods}

A historical cohort study was performed to assess the influence of acute rejection episodes (AREs) on patientgraft survival rates. A thorough review of the files of patients with renal transplanted was conducted between 1990 and 2011 in the renal transplant unit of Taleghani Hospital of Shahid Beheshti University in Tehran, Iran. The same surgical team usually performed surgeries during this period. Naturally donors and recipients were matched based on their age. Hemodialysis was performed 24 hours prior to transplantation. All patients received Cyclosporine (7-8 mg/kg), 500-1000 mg of intravenous methyl prednisolone before the operation, as well a 3-day bolus of intravenous methyl prednisolone therapy after 
the transplantation. A standard triple therapy comprising Cyclosporine A (CsA), Azathioprine (Aza) or Mycophenolate mofetil (MMF), and Prednisone was administrated as primary maintenance immunosuppressive regimen. After the operation, Aza (1.5-2 mg/kg/day) or MMF (1.5-2 g/day) was administered and the dosage was adjusted based on the white blood cell count. CsA (7-8 mg/kg/day) was administered two days after the transplantation when the level of creatinine reduced and the dosage was adjusted according to trough level concentrations. Oral prednisone was subsequently tapered at a daily dosage of $0.6 \mathrm{mg} / \mathrm{kg}$ for one month, after which the daily dosage was tapered to 7.5-5 mg in three months. We defined EAR as a rejection occurred in less than three months and LAR as a rejection happened after the first 3-6 months. Recipients were divided into three groups: Group-1 (no rejection), Group-2 (EAR) and Group-3 (LAR). The degree of allograft function was evaluated by measuring serum creatinine, urea, electrolytes and daily urine output. Any rejection episode was determined through clinical information, color doppler ultrasonography and renal DTPA isotope scan. Bolus methylprednisolone was used as initial therapy for ARE. In case, there was no initial response to corticosteroids, polyclonal or monoclonal antibodies were used as a follow-up therapy. Finally, all patients were followed up routinely at the outpatient center of the hospital. Factors considered to have influence on patient-graft survival were donors' and recipients' age and gender, the type of donor (cadaver, living related and living unrelated), number of transplantations, duration of hemodialysis or peritoneal dialysis and status of panel reactivity antibody. After gathering information, the transplanted patients-grafts data were divided into four groups of active, graft loss, deaths and inconclusive. Finally, based on the collected information, patient-graft survival rates were calculated. Graft loss was defined as the need for resumption of dialysis or patient dying due to any reason, even though the graft was functioning.

\subsection{Statistical Analysis}

All statistical analyses were performed using SPSS software for windows (version 16.0). The overall patient-graft survival was calculated for each group using the KaplanMeier method. Survival duration was compared between the groups by Log-Rank test. Means of quantitative variables were compared using student t-test between the two groups and ANOVA test among the three groups, while Chi-square test was applied for discontinuous variables.

\section{Results}

We reviewed data of 663 kidney transplant cases, performed between 1990 and 2011 at Taleghani Hospital. There were 407 (61.4\%) male and 256 (38.6\%) female recipients, whose mean age was $34.87 \pm 12.96$ years. As for the donors, 526 (79.3\%) of them were males and $137(20.7 \%)$ females, whose mean age was $28.87 \pm 7.03$ years. The mean of recipients' dialysis duration was $18.75 \pm 13.78$ months. Panel reactivity antibody had positive results in five patients (0.75\%) and negative in 658 patients (99.25\%). The type of transplantations was as follows: living related 93 cases (14\%), living unrelated 550 cases (82.9\%), cadaver 15 (2.3\%) and inconclusive data 5 (2\%). Of 663 patients, 638 patients were first time transplant recipients, 23 of them were second time recipients and two patients were inclusion data transplant recipients. Demographic characteristics of patients and donors along with other pretransplantation patients' data are shown in Table 1. Table 1 also shows groups rejection episodes. Only four patients were evaluated by kidney biopsy and the rest were diagnosed by renal scanning, measuring creatinine level and observing clinical symptoms.

Among the variables listed in Table 1, the recipients' gender and donors' age were more influential. LAR was associated with male recipients $(\mathrm{P}=0.001)$ and donors' age with higher than mean age had association with rejection $(\mathrm{P}=$

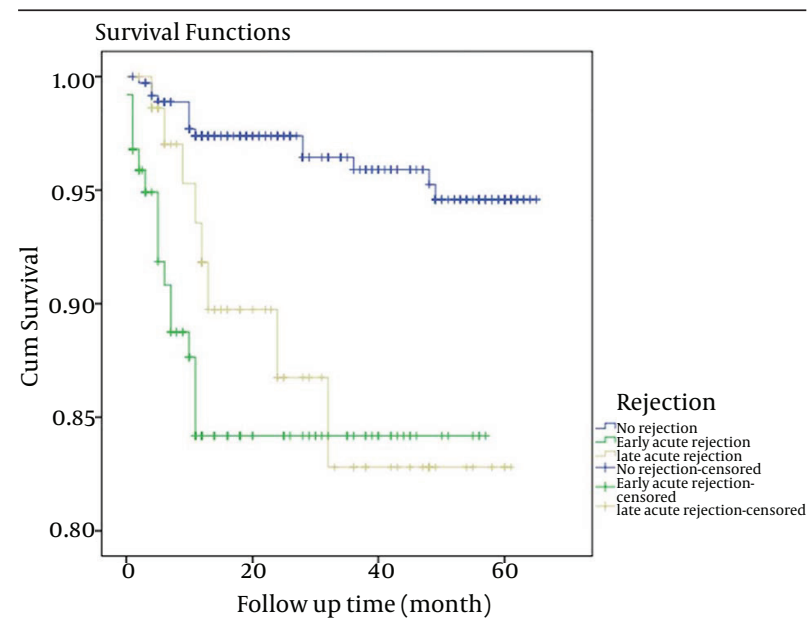

Figure 1. Kaplan-Meier Patient Survival Curves for Patients Considering Rejection Episodes in Five Years

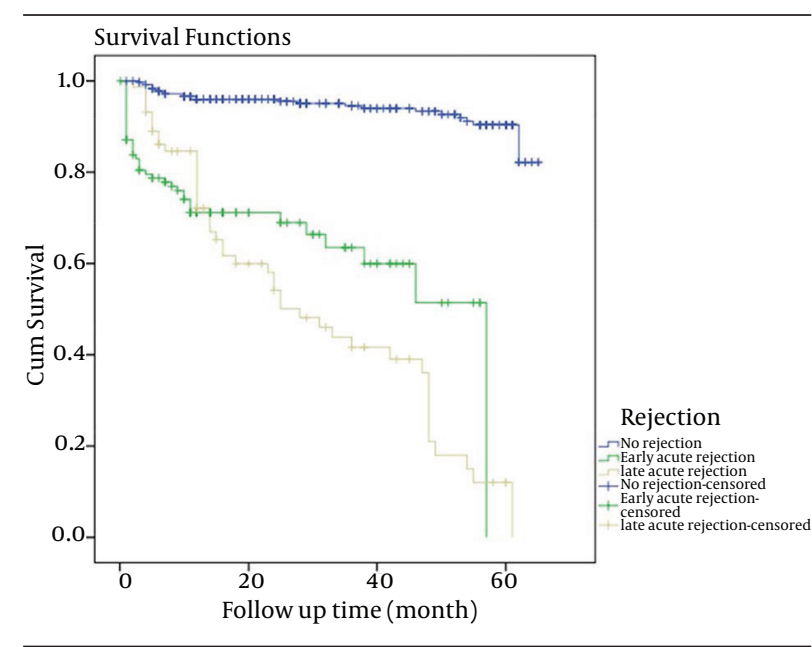

Figure 2. Kaplan-Meier Graft Survival Curves for Patient Considering Rejection Episodes in Five Years 
0.0002). The overall patient survival rates of one and five years after renal transplantation were $94.87 \%$ and $93.8 \%$, respectively; while, the overall graft survival rates of one and five years were $92.6 \%$ and $81.9 \%$, respectively. The incidence of rejection episodes were as follows; 461 (69.5\%) had no rejection, 125 (18.8\%) developed EAR and 77 (11.7\%) had LAR. The Group-1 patient survival rate of one and five years were $97.4 \%$ and $96.8 \%$, for Group-2 were $85.6 \%$ and $84 \%$, and for Group-3 were $92.2 \%$ and $89.4 \%$, respectively as shown in Table 2. Graft survival rates at one and five years for Group-1 after transplantation were $96.7 \%$ and $94.5 \%, 72 \%$ and $61 \%$ for Group-2 and $84.4 \%$ and 36.8\% for Group-3, respectively as demonstrated in Table 3. Patient survival rates showed a slight trend to higher values in Group-1 at one and five years as presented in Figure 1. As shown in Figure 2, fiveyear graft survival rates were higher for Group-1 with significance statistical difference compared to the other two groups ( $\mathrm{P}=<0.0001)$. The five-year graft survival decreased significantly in Group-3 ( $\mathrm{P}=0.0001)$. In Group-1, graft survival was higher than the other two groups. Cox regression model revealed that the age and gender of donors and recipients and the type of transplant were not effective factors in patients and graft survival rate in renal transplants. However, creatinine level at the time of discharge from hospital was an effective factor in patients and graft survival rate as shown in Table 4 .

\begin{tabular}{|c|c|c|c|c|}
\hline Variables & No Rejection Rate & Early Acute Rejection Rate & Late Acute Rejection Rate & Results \\
\hline Recipients gender (male/female) & $287 / 174(62.3)$ & $62 / 63(49.6)$ & $58 / 19(75.3)$ & 0.001 \\
\hline Recipients age & $34.89 \pm 13.38$ & $33.36 \pm 10.27$ & $30.45 \pm 9.37$ & NS \\
\hline Duration of dialysis & $19.53 \pm 14.6$ & $31.98 \pm 18.46$ & $16.86 \pm 11.09$ & $<0001$ \\
\hline PRA status (+/-) & $4 / 457(0.9)$ & $1 / 124(0 / 1)$ & $0 / 77(0)$ & NS \\
\hline $\begin{array}{l}\text { Number of transplantation, (first/sec- } \\
\text { ond/inconclusive) }\end{array}$ & $441 / 18 / 2(96.1)$ & $124 / 1(99.2)$ & $73 / 4(94.8)$ & NS \\
\hline Source of transplanted organ & & & & NS \\
\hline Living related & $62(13.6)$ & $16(12.9)$ & $15(19.5)$ & \\
\hline Living unrelated & $384(84)$ & $105(84.7)$ & $61(79.5)$ & \\
\hline Cadaver & $11(2.4)$ & $3(2.4)$ & $1(1.3)$ & \\
\hline Inconclusive & 4 & 1 & 0 & \\
\hline Donor gender & & & & NS \\
\hline Male & 372 & 99 & 55 & \\
\hline Female & 89 & 26 & 22 & \\
\hline Donor age & $28.21 \pm 6.4$ & $29.91 \pm 7.41$ & $30.5 \pm 8.8$ & 0.0002 \\
\hline Serum cr. at discharge, $\mathrm{mg} / \mathrm{dL}$ & $1.29 \pm 0.44$ & $2.55 \pm 2.25$ & $1.67 \pm 1.27$ & $<0.0001$ \\
\hline \multicolumn{5}{|l|}{ 5-Year outcome } \\
\hline Active & $400(91.3)$ & $45(45)$ & $20(26.3)$ & $<0.0001$ \\
\hline Graft loss & $24(5.5)$ & $39(39)$ & $48(63.2)$ & \\
\hline Death & $14(3.2)$ & $16(16)$ & $8(10.5)$ & \\
\hline Inconclusive & 23 & 25 & 1 & \\
\hline
\end{tabular}

\begin{tabular}{|c|c|c|c|c|}
\hline & No Rejection Rate & Early Acute Rejection Rate & Late Acute Rejection Rate & P Value \\
\hline 1-Year & $449 / 461(97.4)$ & $107 / 125(85.6)$ & $71 / 77(92.2)$ & \\
\hline 5-Year & $424 / 438(96.8)$ & $84 / 100(84)$ & $68 / 76(89.4)$ & $<0.0001$ \\
\hline
\end{tabular}

\begin{tabular}{lcccc}
\hline \multicolumn{5}{l}{ Table 3. One Year and 5-Year Graft Survival Rates in Three Groups of Rejection Episodes ${ }^{\text {a }}$} \\
\hline & No Rejection Rate & Early Acute Rejection Rate & Late Acute Rejection Rate & P Value \\
\hline 1-Year & $446 / 461(96.7)$ & $90 / 125(72)$ & $65 / 77(84.4)$ & \\
5-Year & $414 / 438(94.5)$ & $61 / 100(61)$ & $28 / 76(36.8)$ & $<0.0001$ \\
\hline
\end{tabular}

\footnotetext{
${ }^{\mathrm{a}}$ Data are presented as No.(\%).
} 
Jalalzadeh $\mathrm{M}$ et al.

\begin{tabular}{lcccccc}
\hline Table 4. Cox Regression Analysis of Patients and Graft Survival Rate & & & \\
\hline Variables & \multicolumn{3}{c}{ Univariate Analysis } & & Multivariate Analysis \\
\cline { 2 - 7 } & HR & Confidence Interval 95\% & P & HR & Confidence Interval 95\% & P \\
\hline Five-year Patient's Survival & & & & & \\
Recipient gender & 1.06 & $0.42-2.65$ & 0.89 & 1.21 & $0.48-3.05$ & 0.67 \\
\hline Recipient age & 1.02 & $0.69-1.49$ & 0.91 & 1.06 & $0.71-1.58$ & 0.77 \\
\hline Donor gender & 1.86 & $0.75-5.2$ & 0.16 & 3.36 & $1.21-9.3$ & 0.19 \\
Donor age & 2.07 & $0.77-5.5$ & 0.15 & 2.72 & $0.92-8.1$ & 0.06 \\
\hline Creatinine level at discharge & 3.3 & $1.9-5.7$ & 0.001 & 3.7 & $2.1-6.4$ & 0.001 \\
\hline Type of transplant & 1.04 & $0.6-1.7$ & 0.86 & 0.2 & $0.2-1.9$ & 0.17 \\
Five-year graft Survival & & & & & $0.74-2.1$ & 0.39 \\
\hline Recipient gender & 0.95 & $0.76-1.2$ & 0.65 & 1.25 & $0.74-1.2$ & 0.59 \\
\hline Recipient age & 1.02 & $0.69-1.5$ & 0.91 & 0.94 & $0.48-2.0$ & 0.98 \\
\hline Donor gender & 0.99 & $0.55-1.9$ & 0.98 & 0.99 & $0.66-1.59$ & 0.91 \\
\hline Donor age & 0.86 & $0.59-1.25$ & 0.43 & 1.02 & $3.12-5.65$ & 0.001 \\
\hline Creatinine level at discharge & 4.1 & $3.07-5.53$ & 0.001 & 4.2 & $0.38-1.97$ & 0.73 \\
\hline Type of transplant & 1.09 & $0.56-2.11$ & 0.73 & 0.87 & & \\
\hline
\end{tabular}

\section{Discussion}

Following transplantation, recipients are at the risk of AREs. Patients' survival was significantly affected by the combination of DGF and rejection $(6,10,11)$. Long-term survival of renal allograft is significantly associated with AREs (12). Some studies indicated that DGF appears to be a stronger predictive factor for poor graft survival than AR in the first 90 days of post-transplantation (13). Boom et al. (8) argued that although DGF is a risk factor of AR and for suboptimal function at one-year duration, it does not appear to increase the rate of graft loss. AREs had no adverse effect on long-term renal graft function if they were successfully treated (14). In the recent years, the incidence of AREs has decreased due to introduction of newer immunosuppressive medications (15). The main factors for a more successful long-term allograft outcome are immunologic such as low rate of ARE and advancement of immunosuppressant agents $(16,17)$. However, new immunosuppressive agents have little effect on chronic graft dysfunction and long-term renal allograft survival is still a concern (18). Recent research has confirmed that with new immunosuppressive therapy, an EAR is no longer a risk factor of long-term graft failure and chronic allograft nephropathy (19). In preventing severe AR in SPK(Simultaneous pancreas-kidne) transplant patients, Tacrolimus-based immunosuppressive therapy has shown significant advantage over Cyclosporine (20). Today, AR is still a critical risk factor of late transplant failure despite a decrease in occurrence of acute rejections compared to the past (21). In this study, the incidence of rejection episodes was as follows; $69.5 \%$ had no rejection, $18.8 \%$ developed EAR and $11.7 \%$ had LAR. Some previous studies argued that EARs are as much of a risk factor as LARs in chronic graft loss $(22,23)$. In contrast, another re- search showed that only LARs have adverse effect on longterm graft function (24). Our study showed that EARs were not as significant risk factors of chronic graft loss compared with LARs. Both the UNOS and the U.S. Renal Data System databases support this finding (25). However, EAR does not appear to be a risk factor of long-term successful results, it is still a major complication that increases hospital costs. Studies have shown that the number of ARE has negative impact on graft survival $(26,27)$. Furthermore, recipients with one ARE have been found to show better survival rates than those with two or more episodes of rejection (27). The degree of renal damage is estimated by the difference in serum creatinine before and after AREs, which affect allograft survival (28). As discussed earlier, many factors affect patient-graft survival (2-5). In our study, among those variables, gender of recipients and age of donor had some influence. Late acute rejection was associated with male recipients $(\mathrm{P}=0.001)$, while donors with a higher mean age showed association with rejection $(\mathrm{P}=0.0002)$.

The purposes of the present study were to evaluate the patient-graft survival rates in renal transplantation at our center and to analyze different factors that might have affected patient-graft survival in the sample population with an emphasis on rejection. This study showed that the timing of an acute rejection episode was associated with the success rate of patient-graft survival. Late acute rejection appears to reduce long-term allograft survival rate. Overall, one and five-year outcomes of graft survival were affected by graft rejection. The highest amount of graft loss occurred in the late acute rejection group ( $P$ $<0.0001)$ and the best graft survival rates related to patients who did not have acute rejection during the first 
year after transplantation. In conclusion, according to this study, late acute rejection episodes are significant predictive factors for the failure of graft survival.

\section{Acknowledgements}

We would like to thank the Transplant Department of Taleghani Hospital, Shahid Beheshti University, also Mohammad Karimi who helped us in writing the paper.

\section{Authors' Contributions}

Study concept and design: Dr. Mojgan Jalalzadeh; Acquisition of data: Dr. Mojgan Jalalzadeh, Dr. Said Peyrovi; Analysis and interpretation of data: Dr. Mojgan Jalalzadeh, Dr. Nouraddin Mousavinasab; Drafting of the manuscript: Dr. Mojgan Jalalzadeh; Critical revision of the manuscript for important intellectual content: Dr. Mojgan Jalalzadeh, Dr. Nouraddin Mousavinasab, Dr. Said Peyrovi; Statistical analysis: Dr. Nouraddin Mousavinasab.

\section{References}

1. Fiebiger W, Mitterbauer C, Oberbauer R. Health-related quality of life outcomes after kidney transplantation. Health Qual Life Outcomes. 2004;2:2.

2. Tian YF, Liao CH, Chen MJ. Risk factors among donor characteristics which affect graft outcome in paired kidney transplantation. Transplant Proc. 2008;40(7):2281-4.

3. Zhang L, Ma LL, Ma BR, Tian Y. [Effects of donor age and gender on early acute rejection episode in living related donor kidney transplantation]. Zhonghua Yi Xue Za Zhi. 2008;88(48):3407-10.

4. Gjertson DW. Impact of delayed graft function and acute rejection on kidney graft survival. Clin Transpl. 2000:467-80.

5. Toma H, Tanabe K, Tokumoto T, Shimizu T, Shimmura H. Timedependent risk factors influencing the long-term outcome in living renal allografts: donor age is a crucial risk factor for longterm graft survival more than 5 years after transplantation. Transplantation. 2001;72(5):940-7.

6. Harada KM, Mandia-Sampaio EL, de Sandes-Freitas TV, Felipe CR, Park SI, Pinheiro-Machado PG, et al. Risk factors associated with graft loss and patient survival after kidney transplantation. Transplant Proc. 2009;41(9):3667-70.

7. Tesi RJ, Henry ML, Elkammas EA, Ferguson RM. Predictors of longterm primary cadaveric renal transplant survival. Clin Transpl. 1993;7:345.

8. Boom H, Mallat MJ, de Fijter JW, Zwinderman AH, Paul LC. Delayed graft function influences renal function but not survival. Transplant Proc. 2001;33(1-2):1291.

9. Tsai MK, Wu FL, Lai IR, Lee CY, Hu RH, Lee PH. Decreased acute rejection and improved renal allograft survival using sirolimus and low-dose calcineurin inhibitors without induction therapy. Int J Artif Organs. 2009;32(6):371-80.

10. Tapiawala SN, Tinckam KJ, Cardella CJ, Schiff J, Cattran DC, Cole $\mathrm{EH}$, et al. Delayed graft function and the risk for death with a functioning graft. J Am Soc Nephrol. 2010;21(1):153-61.

11. Lai Q, Pretagostini R, Poli L, Levi Sandri GB, Melandro F, Grieco $\mathrm{M}$, et al. Early urine output predicts graft survival after kidney transplantation. Transplant Proc. 2010;42(4):1090-2.

12. Wu J, Chen J, Wang Y, Zhang J, Zhu Z, Shou Z, et al. Impact of acute rejection episodes on long-term renal allograft survival. Chin Med J (Engl). 2003;116(11):1741-5.

13. Jung GO, Yoon MR, Kim SJ, Sin MJ, Kim EY, Moon JI, et al. The risk factors of delayed graft function and comparison of clinical outcomes after deceased donor kidney transplantation: singlecenter study. Transplant Proc. 2010;42(3):705-9.

14. Burke G, 3rd., Ciancio G, Figueiro J, Olson L, Gomez C, Rosen A, et al. Can acute rejection be prevented in SPK transplantation? Transplant Proc. 2002;34(5):1913-4.

15. Hamida FB, Barbouch S, Bardi R, Helal I, Kaaroud H, Fatma LB, et al. Acute rejection episodes after kidney transplantation. Saudi J Kidney Dis Transpl. 2009;20(3):370-4.

16. Wang D, Xu TZ, Chen JH, Wu WZ, Yang SL, Lin WH, et al. Factors influencing second renal allograft survival: a single center experience in China. Transpl Immunol. 2009;20(3):150-4.

17. Kuypers DR. Immunosuppressive drug therapy and subclinical acute renal allograft rejection: impact and effect. Transplantation. 2008;85(7 Suppl):S25-30.

18. Pascual M, Theruvath T, Kawai T, Tolkoff-Rubin N, Cosimi AB. Strategies to improve long-term outcomes after renal transplantation. N Engl J Med. 2002;346(8):580-90.

19. Sijpkens YW, Doxiadis II, Mallat MJ, de Fijter JW, Bruijn JA, Claas $\mathrm{FH}$, et al. Early versus late acute rejection episodes in renal transplantation. Transplantation. 2003;75(2):204-8.

20. Arbogast H, Malaise J, Illner WD, Tarabichi A, Dieterle C, Landgraf $\mathrm{R}$, et al. Rejection after simultaneous pancreas-kidney transplantation. Nephrol Dial Transplant. 2005;20 Suppl 2:ii11-7.

21. Pallardo Mateu LM, Sancho Calabuig A, Capdevila Plaza L, Franco Esteve A. Acute rejection and late renal transplant failure: risk factors and prognosis. Nephrol Dial Transplant. 2004;19 Suppl 3:iii38-42.

22. Marcen R, Morales JM, Fernandez-Rodriguez A, Capdevila L, Pallardo L, Plaza JJ, et al. Long-term graft function changes in kidney transplant recipients. NDT Plus. 2010;3(Suppl_2):ii2-8.

23. Heldal K, Hartmann A, Leivestad T, Svendsen MV, Foss A, Lien B, et al. Clinical outcomes in elderly kidney transplant recipients are related to acute rejection episodes rather than pretransplant comorbidity. Transplantation. 2009;87(7):1045-51.

24. Meier-Kriesche HU, Steffen BJ, Hochberg AM, Gordon RD, Liebman MN, Morris JA, et al. Long-Term Use of Mycophenolate Mofetil is Associated With a Reduction in the Incidence and Risk of Late Rejection. Am J Transplant. 2003;3(1):68-73.

25. Hwang AH, Cho YW, Cicciarelli J, Mentser M, Iwaki Y, Hardy BE. Risk factors for short- and long-term survival of primary cadaveric renal allografts in pediatric recipients: a UNOS analysis. Transplantation. 2005;80(4):466-70.

26. Berczi C, Asztalos L, Kincses Z, Locsey L, Balazs G. Effect of acute rejection episodes on long-term renal graft survival. Transplant Proc. 1998;30(5):1775.

27. Dickenmann MJ, Nickeleit V, Tsinalis D, Gurke L, Mihatsch MJ, Thiel G. Why do kidney grafts fail?A long-term single-center experience. Transpl Int. 2002;15(9-10):508-14.

28. Yates PJ, Nicholson ML. The aetiology and pathogenesis of chronic allograft nephropathy. Transpl Immunol. 2006;16(3-4):148-57. 